\title{
Facing Up to the Question of Ethics in Mindfulness-Based Interventions
}

\author{
Jake H. Davis
}

Published online: 18 December 2014

(C) Springer Science+Business Media New York 2014

\section{Introduction}

Monteiro, Musten and Compson (2015) have highlighted three important areas for assessing contemporary mindfulness-based interventions (MBIs) in light of traditional Buddhist conceptions. First is the adherence of contemporary presentations to fundamental elements of "right mindfulness". The second question is whether the practices taught in MBIs lead to insight into the roots of suffering or instead offer only more symptomatic relief. A third area of critique regards whether the presentation of MBIs should explicitly include the ethical context in which mindfulness is traditionally taught. Each of these areas is crucial for understanding both the potential and also the pitfalls for the modern development of MBIs. I suggest that questions about whether MBIs ought to adhere to Buddhist visions of right mindfulness and of ethical action can only be answered, if at all, as part of a continuing dialogue on the very general ethical question of how it is best for a human being to be. However, questions about the causes of suffering and how to most effectively address them may serve as an area of common concern across human cultures that can be appealed to as a basis for such dialogues on ethics.

A number of the articles in the recent issue of Contemporary Buddhism (January, 2011) have contrasted various Buddhist textual characterizations of right mindfulness with contemporary presentations of mindfulness as non-judgmental present-centered attention. In these debates, it can often sound as if the definition of right mindfulness (Pāli sammā-sati) is fixed by the tradition(s) and the question is whether contemporary presentations in various MBIs adhere to this authoritative definition. However, it is important to recognize that in

J. H. Davis $(\bowtie)$

Department of Psychiatry and Human Behavior, Brown University, Providence, RI, USA

e-mail: jhdavis@vijja.net the context of mindfulness teachings, both ancient and contemporary, the notion of right mindfulness functions first and foremost as an ethical term. This is true quite aside from points about whether sammā is best translated as "right". Instead, the point is that both religious and secular teachers of mindfulness use the term "mindfulness" to present aspects of the vision of human flourishing that they and their own cultural tradition endorse. If one thinks that the development of a nonjudgmental present-centered attention is one of the most valuable ways for human beings to develop themselves, then that will play a central role in one's presentation of mindfulness practice. On the other hand, some individuals and practice traditions might hold instead that such a non-judgmental present-centered attention is not sufficient, or perhaps not even necessary, for a human being to cultivate what is most valuable. If so, their textual and oral presentations of mindfulness practice are likely to stress other aspects, such as analytical and evaluative contemplative practices.

The controversy over the possibility of a mindful sniper, noted by Monteiro et al., exemplifies the breadth of ethical vision that is at stake in defining right mindfulness. To say that the mindfulness of a sniper does not amount to right mindfulness is to say that human beings ought to cultivate other qualities instead of or (more likely) in addition to the kinds of focused attention that are important for being an effective sniper. For instance, it might be that cultivating a more openmonitoring style of attention training would result in feeling more fully the pain involved in the embodied motivation to cause harm, and thus would be incompatible with being a soldier. Indeed, the comprehensiveness of what is at stake in defining mindfulness is explicit in Kabat-Zinn's (2011, p. 290) recent remark that in certain contexts, he has used the term mindfulness as a placeholder for a "universal dharma that is co-extensive, if not identical, with the teachings of the Buddha, the Buddhadharma." Of course, it should not be naively assumed that different practice traditions all lead to the same 
universal goal, path, or truth. Different individuals, different practice traditions, and different cultures hold different visions of how it is best for a human being to be. And if so, it should not be surprising that presentations of mindfulness will vary within traditional contexts, as well as between traditional and modern contexts. Even if we did have access to the Buddha's own words on what is to count as right mindfulness, there is hardly a cross-cultural consensus that we ought to accept the Buddha as the authority on how a human being ought to be. No appeal to authority will settle the question of whether MBIs adhere to right mindfulness, precisely because what is at issue is which qualities and understandings are the ones that a human being ought to cultivate. To settle that question would require nothing short of an answer to the ethical question of how it is best for a human being to be.

The Pāli Nikāyas do present a relatively consistent vision of how a human being ought to be. For one, as a whole, these discourses are very clear that we ought to purify ourselves of greed, hatred, and delusion. Such Buddhist textual claims about which motivational states are to be cultivated, and which are not, do seem to be intended as universal ethical claims, or at least as applicable to all human beings. Monteiro et al. noted this point, citing scholars such as Gombrich and Keown, and went on to draw out an implication for the inclusion of ethics in MBI pedagogy.

...because intentions are either virtuous or not, they cannot differ from one social group to another. Applying this idea to an MBI program, it suggests that the inclusion of an ethical framework in the form of ethical intentions likely would pose no threat to the social or religious identification that participants may hold.

However, from the fact that Buddhist texts claim that their own ethical judgments of various motivational states are universally correct, it hardly follows that Buddhist ethical judgments are indeed universally correct! Many other cultural traditions would also claim universality for their own (very different) visions of how human beings out to be. Monteiro et al. conceded that different behavioral manifestations of the universal value of respect, say, might result in conflicts over "religious and cultural differences in attire". However, the problem is much larger than this. Two cultures might both value compassion, and yet in one of these cultures, maintaining a family's honor takes precedence, resulting in practices such as "honor killings". Likewise, some modern liberals might value righteous anger towards injustice, whereas many traditional Buddhists will see this as misguided.

To impose a pedagogy based implicitly on a Buddhist vision of how human beings should be onto those who do not already accept such a vision may itself be ethically problematic. So there is a strong argument to be made against MBIs simply laying out Buddhist precepts as the correct context within which to train in mindfulness, without some dialogue on the question of whether these are the best ethical guidelines to adopt, and, if so, why. At a minimum, this might involve dialogical practices of clarifying the values that individual students bring to an MBI course. In practice, such explicit clarification in conjunction with explicit strategies for strengthening individuals' resolve to embody the values they already hold may be sufficient to allay many of the concerns noted by Monteiro et al. Nonetheless, from some traditional Buddhist perspectives, such an approach will appear insufficient. In part, this is because contemporary individuals' values, even once they have been clarified, will likely differ in some respects from various traditional Buddhist perspectives. However, as noted above in the context of discussions of right mindfulness, traditional Buddhist ethical visions differ in ways large and small from one another, as well. Moreover, as guides for us today, even the most textually and historically rigorous presentations of traditional concepts of right mindfulness and of right action can at best be taken as suggestions for further inquiry. We can decide to defer to others (our own cultural lineage, a teacher from an exotic tradition) for answers to question about how best to live, but that decision to defer is itself a decision, and as such subject to reflective scrutiny.

The problem of differing visions of human flourishing is raised also by the further critique that secular applications of mindfulness may provide only symptomatic relief of suffering. From many traditional perspectives, the ultimate goal of mindfulness practice is to achieve liberation from the cycle of rebirth. MBIs are not presented in a context in which that specifically Buddhist metaphysical vision can be assumed, and so they cannot realistically hope to motivate (many of) their students by appealing to the prospect of liberation from rebirth. However, the Buddhist ideal of realizing the cessation of anguish and unease - dukkha - may in fact provide a note of consilience between traditional and secular presentations. To the degree that some specific traditional conception does not involve eliminating all kinds of dukkha in this very life, then to that degree, neither can MBIs be expected to achieve that. Conversely, to the degree that the cession of anguish and unease is something that can in principle be achieved in a human life, through traditional practices of training universally human cognitive faculties, then it is not clear why MBI interventions involving training attention in the right ways could not, in principle, achieve the same goal. Of course, many of us in a secular Western context may be sufficiently content with a reduction of some symptoms of dukkha that we do not persevere on to address its deepest roots in the mind. On the other hand, that seems to be the case for many traditional Buddhists as well, the metaphysical visions of rebirth and liberation they may hold notwithstanding.

The most important and most challenging critique of mindfulness teachings in our contemporary context, to my mind, is 
one that applies equally to traditional Buddhist presentations as well as to modern MBIs. The challenge in both arenas is for teachers to justify their (different) ends as well as their (different) means, in the context of a cross-cultural dialogue about how it is best for human beings to be, by appealing to considerations that have force for all human beings. Arguably, the aim to most effectively reduce anguish and unease can provide just such a universal starting point for conversations focused on how human beings ought to be motivated. One way to formulate such a proposal is to evaluate actions on the basis of whether they reduce aggregate suffering in the world. However, a different approach more in line with the Pāli Nikāyas is to focus on which sorts of motivational states are characterized by ease, and which by unease, for the agents themselves (Davis, forthcoming). Nonetheless, the point should not be to establish as true some one traditional Buddhist vision of ethics. Rather, questions about the transmission of mindfulness across cultural contexts make clear the need to engage in a dialogue that can challenge all of us, from every cultural tradition, to carefully examine our deepest convictions about how it is best for human beings to be, and thus how it is best for each of us to train our minds.

\section{References}

Kabat-Zinn, J. (2011). Some reflections on the origins of MBSR, skillful means, and the trouble with maps. Contemporary Buddhism, 12(1), 281-306. doi:10.1080/14639947.2011.564844.

Monteiro, L. M., Musten, R. F., \& Compson, J. (2015). Traditional and contemporary mindfulness: finding the middle path in the tangle of concerns. Mindfulness, 6, 1-13. 